\title{
SUPERSIMPLE FIELDS AND DIVISION RINGS
}

\author{
A. Pillay, T. Scanlon, and F. O. Wagner \\ ABstract. It is proved that any supersimple field has trivial Brauer group, and \\ more generally that any supersimple division ring is commutative. As prereq- \\ uisites we prove several results about generic types in groups and fields whose \\ theory is simple.
}

\section{Introduction}

Simple theories were introduced by Shelah in [12]. In [3], Kim, continuing Shelah's work, showed how the theory of forking transfers almost completely from the stable context to the more general simple context. In [4] the "Independence Theorem" was proved for simple theories, giving a satisfactory analogue of the theory of stationarity from stability theory.

In [8], Poizat's theory ([10]) of generic types and stabilizers in stable groups was generalized to the case of groups definable in simple theories. Further generalizations appear in [13].

Stability-theoretic algebra studied, among other things, the algebraic consequences of imposing stability-theoretic conditions on a group, ring or field. Among the main results in the area was the Macintyre-Cherlin-Shelah theorem ([7], [1]) saying that any infinite field whose theory is superstable must be algebraically closed.

Supersimple fields (namely fields with supersimple theory) on the other hand form a broader class. In [5] it is shown that any perfect pseudo-algebraically closed $(P A C)$ field with "small" absolute Galois group is supersimple; in fact of $S U$-rank 1 in the language of rings. (Ultraproducts of finite fields furnish examples.) The work in this paper is partly motivated by the conjecture that these are the only cases. It follows from [9] that any supersimple field is perfect and has small absolute Galois group. In the present paper we show that a supersimple field $K$ has trivial Brauer group. As supersimplicity is preserved under finite extensions, this is, by [11], X.Prop.11, equivalent to the norm map $N_{L / K}: L^{*} \rightarrow K^{*}$ being surjective for any finite Galois extension $L$ of $K$. Triviality of the Brauer group also implies that any rational variety over $K$ has a $K$-rational point, yielding in a sense a first approximation to the conjecture that any supersimple field must be $P A C$.

Received March 24, 1998.

The first author was partially supported by NSF grant DMS 96-96268.

The second author was supported by an NSF Postdoctoral Fellowship.

The third author was supported by DFG grant Wa899/2-1. 
We assume acquaintance with the notions and machinery from [4] and [8]. In section 2, some additional results are obtained about generic types and connected components of groups in simple theories. In section 3, we show that if $K$ is a field in a simple theory then the notions of additive and multiplicative generic coincide. We also make some observations about the interaction between the additive and multiplicative connected components. These results are applied in section 4 to show triviality of the Brauer group in the supersimple case. Some additional algebraic arguments yield in section 5 commutativity of any supersimple division ring.

\section{More on generic types and stabilizers}

Let us fix a saturated model $\bar{M}$ of a simple theory, and an infinite group $G$ which is type-definable in $\bar{M}$ over $\emptyset$ say. All complete types $p(x)$ we consider will be types of elements of $G$ (namely we assume " $p(x) \rightarrow x \in G$ "). We will often wish to work with Lascar strong types (so that we can use the Independence Theorem). This is most easily accomplished by working with types over models. So $M$ will denote a small elementary substructure of $\bar{M}$, which may vary. $A, B$ as usual denote small subsets of $\bar{M}$.

We briefly recall (from [8]) the notions of generic type, stabilizer, and connected component.

Generics. Let $a \in G$. Then $t p(a / A)$ is (left-) generic (for $G$ ) if whenever $b \in G$ is independent from $a$ over $A$ then $b$. $a$ (the product of $b$ and $a$ ) is independent from $A \cup\{b\}$ over $\emptyset$. (We also say that $a$ is a generic element of $G$ over $A$.) We have associated notions of a (type)-definable subset of $G$ being generic in $G$. Moreover the notions of left-generic and right-generic coincide.

Stabilizers. For $p(x) \in S(M), S t(p)$ is defined to be $\{b \in G$ : for some realization $a$ of $p$ which is independent from $b$ over $M, b . a$ realises $p$ and is independent from $b$ over $M$ \}. St $(p)$ is type-definable over $M$. Let $\operatorname{Stab}(p)$ be the subgroup of $G$ generated by $S t(p)$. Then it turns out that $S t a b(p)$ is type-definable over $M$, and moreover that $S t(p)$ is "large" in $S t a b(p)$ in the sense that any generic element of $\operatorname{Stab}(p)$ over $M$ is in $S t(p)$.

Remark 2.1. One can also define $S t(p, q)$ for $p(x), q(x) \in S(M)$ in the obvious way: $S t(p, q)=\{b \in G$ : for some a realising $p$ such that a is independent from $b$ over $M$, b.a realises $q$ and is independent from $b$ over $M\}$. St $(p, q)$ is again type-definable over $M$ and one sees easily, using the Independence Theorem, that $S t(p, q)$ is, if nonempty, a large subset of a translate of $\operatorname{Stab}(p)$.

Connected components. For any set $A$ of parameters, $G_{A}^{0}$, the connected component of $G$ over $A$, is by definition the smallest type-definable over $A$ subgroup of $G$ of bounded index. A key fact is that $p(x) \in S(M)$ is a generic type of $G$ if and only if $\operatorname{Stab}(p)$ contains $G_{M}^{0}$. Also clearly any generic type of $G_{A}^{0}$ is 
a generic type of $G$. In contrast to the stable situation, the connected component of $G$ over $A$ may vary with $A$. On the other hand $G_{A}^{0}$ is always a normal subgroup of $G$.

If $G$ happens to be stable then we know that $G^{0}$ has a unique generic type, whose stabilizer is $G^{0}$. The next lemma gives an analogue of this in the simple case.

Proposition 2.2. Let $p(x), q(x), r(x) \in S(M)$ be generic types of $G_{M}^{0}$. Then there are realizations $a, b, c$ of $p, q, r$ respectively, which are pairwise $M$-independent, and with $a . b=c$.

Proof. This can be seen using Remark 2.1, but we give a direct proof. Let $b, c$ be independent (over $M$ ) realizations of $q, r$ respectively. Let $a^{\prime}=c . b^{-1}$. Then $a^{\prime} . b=c, a^{\prime}$ is generic in $G_{M}^{0}$ over $M$ and $\left\{a^{\prime}, b, c\right\}$ is pairwise $M$-independent. We can similarly find $d$ generic in $G_{M}^{0}$ over $M$, such that $a=a^{\prime} . d$ realises $p$ and $\left\{d, a^{\prime}, a\right\}$ is pairwise $M$-independent. By the facts above on connected components and generics, $\operatorname{Stab}(q)$ contains $G_{M}^{0}$ and hence any generic element of $G_{M}^{0}$ over $M$ is in $S t(q)$. In particular $d^{-1} \in S t(q)$. By the Independence Theorem we can assume that $\left\{d, a^{\prime}, b\right\}$ is pairwise $M$-independent and that $d^{-1} \cdot b=b^{\prime}$ realises $q$. Then $a \cdot b^{\prime}=\left(a^{\prime} \cdot d\right) \cdot b^{\prime}=a^{\prime} \cdot\left(d \cdot b^{\prime}\right)=c$ and easily $\left\{a, b^{\prime}, c\right\}$ is pairwise $M$-independent.

\section{Simple fields}

In this section $F$ will be an infinite field which is type-definable over $\emptyset$ in the saturated model $\bar{M}$ of a simple theory $T$. Types $p(x), q(x)$ will be types of elements of $F$. We are going to apply Proposition 2.2 in order to understand the interaction between generic additive subgroups and generic multiplicative subgroups in simple fields. (In the stable case the situation is clear: a stable field is connected both additively and multiplicatively [1].) We begin by pointing out that in simple fields additive and multiplicative generics coincide.

Proposition 3.1. Let $F$ be an infinite field which is type-definable in $\bar{M}$ over $\emptyset$. Then a type $p(x) \in S(A)$ is an additive generic type of $F$ iff it is a multiplicative generic type of $F$.

Proof. We begin with

Claim 1. If $p(x) \in S(A)$ is additively generic then for any nonzero $a \in F$ and realisation $c$ of $p$ independent from $a$ over $A, \operatorname{tp}(a . c / A \cup\{a\})$ is an additive generic of $F$.

Proof of Claim 1. Let $a \in F$ and $M$ contain $A \cup\{a\}$. Let $p^{\prime}$ be a nonforking extension of $p$ over $M$, realised by $b$. Then $p^{\prime}$ remains an additive generic of $F$. So the additive stabilizer of $p^{\prime}, \operatorname{Stab}^{+}\left(p^{\prime}\right)$ is a type-definable over $M$ subgroup of $\mathrm{F}^{+}$of bounded index. It follows that $\operatorname{Stab}^{+}\left(a \cdot p^{\prime}\right)=a \cdot \operatorname{Stab}^{+}\left(p^{\prime}\right)$ also has bounded index. By the remarks above on connected components a. $p^{\prime}$ is an additive generic type of $F$. In particular $a . p^{\prime}$ does not fork over $\emptyset$. 
From Claim 1, using the fact that $\operatorname{tp}($ a.c $/ A \cup\{a\})$ there, being an additive generic, does not fork over $\emptyset$, we see that:

Claim 2. Any additive generic type is a multiplicative generic type.

Claim 3. Any multiplicative generic type of $F$ is an additive generic type of $F$.

Proof of Claim 3. Let $q(x) \in S(A)$ be a multiplicative generic type. We may replace $q$ any time by a nonforking extension. By Claim 2 (and the existence of generic types) there is an additive generic type $p(x) \in S(A)$ which is also multiplicatively generic. It follows that there is $c \in F, M$ containing $A \cup\{c\}$ and nonforking extensions $q^{\prime}, p^{\prime}$ of $q, p$ over $M$ such that $c . q^{\prime}=p^{\prime}$. So $c . q^{\prime}$ is an additive generic type. By Claim $1, q^{\prime}$ is also an additive generic type.

Below we will also use $T$ to denote a subgroup of the multiplicative group of $F$. We hope this does not create any confusion as $T$ was used above for a complete theory.

Lemma 3.2. Let $T$ be a multiplicative subgroup of $F$ of bounded index, typedefinable over $A$. Then every nonzero coset a.T of $T$ meets $\left(F^{+}\right)_{A}^{0}$ in a generic set.

Proof. Note that if $B \supset A$, then $\left(F^{+}\right)_{B}^{0}$ is contained in $\left(F^{+}\right)_{A}^{0}$. So we may assume that $A$ is a model $M$ which contains a representative of each coset $c . T$ of $T$ in $F^{*}$. Note that

$\left(^{*}\right)$ For each nonzero $c \in F^{M}, c .\left(F^{+}\right)_{M}^{0}=\left(F^{+}\right)_{M}^{0}$ (as multiplication by $c$ is an additive endomorphism of $F$ which interchanges $M$-definable sets).

Let $p(x)$ be a generic type of $G_{M}^{0}$ over $M$. Let $S$ be a coset of $T$ in $F^{*}$. Then there is $c \in F^{M}$ such that $c . p$ is the type of an element of $S$. By Claim 1 in the proof of Proposition 3.1, together with $(*), c . p$ is a generic type of $\left(F^{+}\right)_{M}^{0}$. This proves the lemma.

Remark 3.3. In fact, with the assumptions of Lemma 3.2, every coset of $\left(F^{+}\right)_{A}^{0}$ in $F^{+}$meets every coset of $T$ in $F^{*}$ in a generic set.

Proof. We may assume that $A$ is a model $M$ which contains representatives of all cosets of $T$ in $F^{*}$. To prove the remark, it is enough (using Lemma 3.2) to show that if $S$ is a coset of $\left(F^{+}\right)_{M}^{0}$ in $F^{+}$then $S \cap T$ is generic. Let $b \in S$ be nonzero. Let $M^{\prime}$ be a model containing $M \cup\{b\}$. By Lemma 3.2, we can find generic $d$ in $\left(F^{+}\right)_{M^{\prime}}^{0}$ over $M^{\prime}$ such that $d \in b^{-1} . T$. Let $c=d-1$. Then $c$ is generic in $\left(F^{+}\right)_{M^{\prime}}^{0}$ over $M^{\prime}$ too, and as in the proof of Lemma 3.2, b.c is generic in $\left(F^{+}\right)_{M^{\prime}}^{0}$ over $M^{\prime}$. So $b . d=b . c+b$ is generic in $S$ over $M$. On the other hand $b . d \in T$. Thus $S \cap T$ is generic, as required.

Proposition 3.4. Let $T$ be any multiplicative subgroup of $F$ of bounded index. Let $S_{1}, S_{2}$ be cosets of $T$ in $F^{*}$. Then $S_{1}+S_{2}$ (the set of $s_{1}+s_{2}$ for $s_{1} \in S_{1}$ and $s_{2} \in S_{2}$ ) contains $F^{*}$. In fact for any nonzero $d \in F$ there are $a, b \in S_{1}, S_{2}$ respectively, each generic over $d$ such that $a+b=d$. 
Proof. Again we work over a reasonably saturated model $M$ (so in particular $T, S_{1}, S_{2}$ are defined over $\left.M\right)$. Let $d \in F^{M}$ be nonzero, and let $S_{3}$ be $d . T$. By Lemma 3.2, let $p(x), q(x), r(x)$ be generic types of $\left(F^{+}\right)_{M}^{0}$ such that $p(x) \rightarrow$ $x \in S_{1}, q(x) \rightarrow x \in S_{2}$ and $r(x) \rightarrow x \in S_{3}$. By Proposition 2.2, there are realisations $a, b, c$ of $p, q, r$ respectively, such that $a+b=c$, where moreover $\{a, b, c\}$ is pairwise independent over $M$. Multiplying on the right by $c^{-1} . d \in T$, we obtain $a^{\prime} \in S_{1}, b^{\prime} \in S_{2}$ with $a^{\prime}+b^{\prime}=d$. Clearly each of $a^{\prime}, b^{\prime}$ are generic over $M$. This completes the proof.

[Here is a sketch of another proof of 3.4, avoiding use of Proposition 2.2 and containing some possibly useful ideas: Work again over a model $M$. Let $X=$ $S t\left(S_{1}, S_{2}\right)$ which by definition equals $\{a \in F$ : for each generic type $p(x) \in S(M)$ of $S_{1}$ there is $c$ realising $p$ independent from $a$ such that $\left.a . c \in S_{2}\right\} . X$ is typedefinable over $M$ and moreover (see 2.2) there is a type-definable set $X^{\prime}$, a union of cosets of $\left(F^{+}\right)_{M}^{0}$ in $F^{+}$such that $X \subset X^{\prime}$ and also every generic element of $X^{\prime}$ over $M$ is in $X$. Note that $X$ is invariant under multiplication by $T$. Let now $H$ be the set-theoretic stabilizer of $X^{\prime} . H$ is a type-definable over $M$ subgroup of $F^{+}$containing $\left(F^{+}\right)_{M}^{0}$. H is also invariant under multiplication by $T$, but we know that $\left(F^{+}\right)_{M}^{0}$ intersects each coset of $T$ in $F^{*}$. Thus $H=F^{+}$. It follows that $X^{\prime}=F$, and thus $X$ contains all generic types over $M$. As $X$ is also invariant under $T$ it follows that $X$ contains $F^{*}$.]

The characteristic 0 case of the following was observed earlier together with Zoe Chatzidakis.

Corollary 3.5. If $F$ is supersimple, then any conic defined over $F$ has an $F$ rational point.

Proof. A conic defined over $F$ can be put into the form $x^{2}+a y^{2}=b$ for some nonzero $a, b \in F$. As we note in the next section, supersimplicity of $F$ implies that the squares form a definable subgroup of $F^{*}$ of finite index. By 3.4, the equation has a solution in $F$.

\section{Triviality of the Brauer group for supersimple fields}

We will prove that if $F$ is an infinite field possibly with extra structure, whose theory is supersimple, then $F$ has trivial Brauer group. We will see in the course of the proof that this is a first order property, so we will assume $F$ to be saturated. We will work with the formalism of the previous section, namely we assume $F$ to be definable over $\emptyset$ in a big model $\bar{M}$ of a supersimple theory.

At this point it is worth mention the relevant facts about the $S U$-rank, most of which will be used in the next section. Each complete type has ordinal valued $S U$-rank, and the generic types of a (type-)definable group $G$ are precisely the types of maximal $S U$-rank in $G$ (which exist). The following is mentioned explicitly in [13]. 
Fact 4.1. (i) For any elements $a, b$ and set $A$ of parameters,

$$
S U(a / A \cup\{b\})+S U(b / A) \leq S U(a, b / A) \leq S U(a / A \cup\{b\}) \oplus S U(b / A) .
$$

(ii) Let $G$ be a type-definable group and $H$ a type-definable subgroup, then

$$
S U(H)+S U(G / H) \leq S U(G) \leq S U(H) \oplus S U(G / H) .
$$

(iii) Suppose $D$ is a type-definable division ring, then $D$ is definable and has monomial SU-rank (namely SU-rank of the form $\omega^{\alpha} . n$ for some ordinal $\alpha$ and some positive integer $n$ ).

The relevant consequences for this section are:

Fact 4.2. (i) for each $n$, the group of nth powers $\left(F^{*}\right)^{n}$ has finite index in $F^{*}$, (ii) $F$ is perfect.

Proof. (i) Let $a \in F$ be generic over $\emptyset$. Let $b=a^{n}$. Then $a \in a c l(b)$, so $S U(a / b)=0$. Thus by Fact 4.1. (i) $S U(a)=S U(b)$, whereby $b$ is generic in $F$ too. Thus $\left(F^{*}\right)^{n}$ is a generic subgroup of $F^{*}$ and has finite index (as it is definable).

(ii) Supposing $F$ to have characteristic $p>0$, note that as in (i) the additive group of $F^{p}$ has finite index in $F$. But $F / F^{p}$ is a vector space over the field $F^{p}$, a contradiction (the latter being infinite) unless $F^{p}=F$.

Note that any finite extension of $F$ is also definable in $\bar{M}$, so Fact 4.2 applies to all finite extensions of $F$.

We will now recall relevant notions and facts concerning the Brauer group, the norm map and Galois cohomology. In fact one can extract a general result which says that for the Brauer group of every finite extension of a field $F$ to be trivial it is enough that for any finite extension $\mathrm{K}$ of $\mathrm{F}$ and Kummer extension $L$ of $K, N_{L / K}$ is surjective. (See Facts 4.3 to 4.5 below. We are assuming that every finite extension of $F$ is perfect.) Proposition 3.4 will allow us to conclude triviality of the Brauer group for supersimple $F$. Rather than simply state this reduction, we will include an explanation of it as part of the proof, which will entail giving some definitions.

We first discuss the Brauer group. The reader can look at [2] and [11] for further details. We will assume that $F$ and all its finite extensions are perfect.

By a central simple algebra over $F$ we mean a finite dimensional $F$-algebra $A$ whose centre is $F$ and which has no nontrivial two-sided ideals. If $A$ and $B$ are two such objects then so is the tensor product $A \otimes_{F} B$. $A$ and $B$ are called equivalent (or similar) if for some $m, n$ the matrix algebras $M_{m}(A), M_{n}(B)$ are isomorphic (as $F$-algebras). The tensor product operation respects this equivalence relation and turns the set of classes into an abelian group. This group is called the Brauer group of $F, B r(F)$, and is an important invariant of the field $F$. Any central simple $F$-algebra $A$ will be a matrix algebra over a certain finite dimensional division algebra $D$ with centre $F$. Moreover the equivalence class of $A$ is determined by and determines the isomorphism type of $D$. The 
trivial element of $\operatorname{Br}(F)$ then corresponds to $F$ itself. On the other hand, for any central simple algebra $A$ over $F$ there is some finite extension $K$ of $F$ such that $A \otimes K$ is isomorphic to a matrix algebra over $K$, hence represents the trivial element of $\operatorname{Br}(K)$. ( $K$ is called a splitting field for $A$.)

If $K$ is a finite extension of $F$ then tensoring with $K$ determines a homomorphism from $\operatorname{Br}(F)$ into $\operatorname{Br}(K)$. The kernel of this homomorphism is denoted $\operatorname{Br}(K / F)$. From the previous paragraph, $\operatorname{Br}(F)$ is the union of all $\operatorname{Br}(K / F)$ as $K$ runs over finite extensions of $F$, in fact, over finite Galois extensions of $F$.

For $K$ a finite Galois extension of $F$ with Galois group $G$, there is a classical isomorphism of $\operatorname{Br}(K / F)$ with the Galois cohomology group $H^{2}\left(G, K^{*}\right)$ : given a 2-cocycle $f: G \times G \rightarrow K^{*}$, define a $F$-algebra structure on the $K$-vector space $A$ with basis $\left\{u_{s}: s \in G\right\}$ by: $u_{s} \cdot u_{t}=f(s, t) u_{s t}$ and for $e \in K, u_{s} e=(s e) u_{s}$. Then $A$ becomes a central simple $F$-algebra $A_{f}$, and the map $f \rightarrow A_{f}$ determines an isomorphism between $H^{2}\left(G, K^{*}\right)$ and $\operatorname{Br}(K / F)$.

We have the following fact (see [11], X.6):

Fact 4.3. Let $F<K<L$ where both $K$ and $L$ are Galois extensions of $F$ with Galois groups $H, G$ respectively. Then there is an exact sequence

$0 \rightarrow H^{2}\left(H, K^{*}\right) \rightarrow H^{2}\left(G, L^{*}\right) \rightarrow H^{2}\left(\operatorname{Gal}(L / K), L^{*}\right)$.

We also need ([11], IX):

Fact 4.4. Let $G$ be a finite group and $A$ a $G$-module. Let $n \geq 1$. Suppose that for all primes $p, H^{n}\left(G_{p}, A\right)=0$ where $G_{p}$ is a Sylow subgroup of $G$. Then $H^{n}(G, A)=0$.

Finally, for $K$ a finite Galois extension of $F$, the norm map $N_{K / F}: K^{*} \rightarrow F^{*}$ is the map which takes any $a \in K^{*}$ to the product of all $\sigma(a)$ where $\sigma$ runs over $\operatorname{Gal}(K / F)$. See Theorem 8.14 of [2] for:

Fact 4.5. Let $K$ be a cyclic extension of $F$ with Galois group $G$. Then $H^{2}\left(G, K^{*}\right)$ is isomorphic to the quotient group $F^{*} / N_{K / F}\left(K^{*}\right)$.

We can now prove:

Theorem 4.6. ( $F$ a supersimple field.) $\operatorname{Br}(F)$ is trivial.

Proof. We will prove by induction on $n$, that for every finite extension $K$ of $F$ and every Galois extension $L$ of $K$ of degree $n, B r(L / K)$ (or equivalently $\left.H^{2}\left(\operatorname{Gal}(L / K), L^{*}\right)\right)$ is trivial. By the remarks above this is enough. Suppose this is proved for all $n<m$ and we want to prove it for $m$. Let $K$ be a finite extension of $F$ and $L$ a Galois extension of $K$ with Galois group $G$ of order $m$. If $G$ has a proper normal subgroup $H$ then let $K_{1}$ be $F i x(H)$. By inductive hypothesis, both $H^{2}\left(H, L^{*}\right)$ and $H^{2}\left(G / H, K_{1}^{*}\right)$ are trivial. By Fact 4.3 , so is $H^{2}\left(G, L^{*}\right)$. So we may assume $G$ to be simple. Now suppose $G$ to be nonabelian (simple). Then the order of $G$ is not a prime power. For each prime $p$ dividing the order of $G$, let $G_{p}$ be a $p$-Sylow subgroup of $G$ and let $K_{p}$ be the fixed 
field of $G_{p}$. By induction hypothesis $H^{2}\left(G_{p}, L^{*}\right)$ is trivial, so by Fact 4.4 so is $H^{2}\left(G, L^{*}\right)$. So we may assume that $G$ is abelian and simple, hence elementary abelian of prime order $p$ say. Let $K_{1}$ be obtained from $K$ by adjoining all $p t h$ roots of unity. Let $L_{1}$ be the compositum of $L$ and $K_{1}$. By Fact 4.3 it is enough to prove that $H^{2}\left(\mathrm{Gal}\left(L_{1} / K\right), L_{1}^{*}\right)$ is trivial. As all prime divisors of the order of $\operatorname{Gal}\left(K_{1} / K\right)$ are strictly less than $p$ it follows by the induction hypothesis, as above, that $H^{2}\left(\operatorname{Gal}\left(K_{1} / K\right), K_{1}^{*}\right)$ is trivial. Thus by Fact 4.3 , it remains to prove only that $H^{2}\left(\operatorname{Gal}\left(L_{1} / K_{1}\right), L_{1}^{*}\right)$ is trivial. Note that $L_{1}$ is an extension of $K_{1}$ of degree $p$.

So, changing notation, we are reduced to showing that $H^{2}\left(\operatorname{Gal}(L / K), L^{*}\right)$ is trivial, when $G=\operatorname{Gal}(L / K)$ has order $p$ and $K$, a finite extension of $F$, contains all $p$ th roots of unity. If $p$ is the characteristic, then by Fact 4.2 (ii), the restriction of $N_{L / K}$ to $K^{*}$ is already surjective, so $N_{L / K}: L^{*} \rightarrow K^{*}$ is surjective, which by Fact 4.5 finishes the proof. So we may assume that $p$ is not the characteristic. In that case, $L$ is a Kummer extension of $K$ generated by a solution $\alpha$ to $x^{p}=a$ for some $a \in K_{1}$. We will show that $N_{L / K}: L^{*} \rightarrow K^{*}$ is surjective.

Note that $\left\{1, \alpha, \alpha^{2}, . ., \alpha^{p-1}\right\}$ is a basis for $L$ over $K$, with respect to which any element of $L$ has coordinates $x_{1}, . ., x_{p}$ from $K$. So the norm map can be represented as a map from the set of $p$-tuples of elements of $K$ (not all zero) to $K^{*}$. Let $\omega$ be a primitive $p t h$ root of unity. Then the conjugates of $\alpha$ under $G$ are $\alpha, \omega \alpha, . ., \omega^{p-1} \alpha$. An easy computation shows that for any $x_{1}, x_{2}$ in $K$, $N_{L / K}\left(x_{1}, x_{2}, 0, \ldots 0\right)=x_{1}^{p}+a x_{2}^{p}$ if $p$ is not 2 and $=x_{1}^{p}-a x_{2}^{p}$ if $p=2$. Now $K$, being a finite extension of $F$ is also supersimple. By Fact 4.2, the multiplicative subgroup $T$ of $K^{*}$ of $p t h$ powers is definable and of finite index. By Proposition 3.4, both $T+a T$ and $T-a T$ contain $K^{*}$. Thus, the image of $N_{L / K}$ must be equal to $K^{*}$. By Fact $4.5, H^{2}\left(G, L^{*}\right)=0$. The proof is complete.

Note that Theorem 4.6 has the following consequence:

Corollary 4.7. Suppose that $F$ is a supersimple field, and $D$ is a finite dimensional division algebra over $F$. Then $D$ is a field.

Proof. By for example 15.8 of [6], if a division ring is finite-dimensional over a subfield then it is finite-dimensional over its centre.

\section{Supersimple division rings}

This section is devoted to a proof of:

Theorem 5.1. Any supersimple division ring is a field.

Corollary 4.7 will play a crucial role in the proof.

We proceed to the proof of Theorem 5.1, which will go through various reductions and cases.

We will assume that $D$ is a supersimple division ring (namely a division ring type-definable in a big model $\bar{M}$ of a supersimple theory), which is not 
commutative, and look for a contradiction. We make continuous use of Fact 4.1. In particular $S U(D)$ is a monomial $\omega^{\alpha} \cdot n$ say.

Now for any finite subset $A$ of $D$, the centralizer of $A$ in $D, C_{D}(A)$ is a definable subdivision ring of $D$. Note that if $D_{1}<D_{2}$ are infinite subdivision rings of $D$ then the index of $D_{1}$ in $D_{2}$ (additively) must be infinite, hence $S U\left(D_{1}\right)<S U\left(D_{2}\right)$. It follows that $D$ has the DCC on centralizers. Now the centre of $D$ is a field. So choosing a smallest centralizer which is not a field, we may make:

Assumption. For every $a \in D \backslash Z(D), C_{D}(a)$ is a field.

Lemma 5.2. $S U(Z(D))<\omega^{\alpha}$ and also $S U\left(C_{D}(a)\right)<\omega^{\alpha}$ for each $a \notin Z(D)$.

Proof. Otherwise, $D$ has finite dimension over a subfield, contradicting Corollary 4.7.

Lemma 5.3. (i) For any $a \notin Z(D)$ the conjugacy class of $a$ in $D, a^{D}$ is generic, and moreover there are only finitely many such conjugacy classes.

(ii) Also for a $\notin Z(D),\left\{x^{a}-x: x \in D\right\}$ is an additive subgroup of finite index of $D$.

Proof. (i) For $a \notin Z(D), a^{D}$ is in definable bijection with $D^{*} / C_{D}(a)^{*}$ which by Fact 4.1 and Lemma 5.2 has $S U$-rank $\omega^{\alpha} . n$. Thus $a^{D}$ is generic in $D$. As the relation of being in the same conjugacy class is an equivalence relation, there can be at most finitely many such generic classes.

(ii) Note that $C_{D}(a)$ is precisely the kernel of the additive endomorphism $\mu$ of $D$ which takes $x$ to $x^{a}-x$. So by Lemma 5.2, $S U(\operatorname{Ker}(\mu))<\omega^{\alpha}$. Fact 4.1 implies that $\operatorname{Im}(\mu)$ has $S U$-rank $\omega^{\alpha} . n$, so has finite index.

Lemma 5.4. If $a, b \notin Z(D)$ do not commute, then $C_{D}(a) \cap C_{D}(b)=Z(D)$.

Proof. Let $c \in C_{D}(a) \cap C_{D}(b)$. If $c \notin Z(D)$ then by the Assumption above, $C_{D}(c)$ is a field, so $a$ and $b$ commute, contradiction.

Lemma 5.5. Assume that $\operatorname{char}(D)=0$. Then

(i) For any $b \in D \backslash Z(D)$ and $n>0, b^{n} \notin Z(D)$

(ii) For all $a \in D \backslash Z(D), a^{D}-a \supseteq Z(D)$.

Proof. (i) Note that as $\operatorname{char}(D)=0, D$ has no additive subgroups of finite index, and thus by Lemma 5.3 (ii), $\left\{x^{b}-x: x \in D\right\}=D$. Thus there are nonzero $c \in Z(D)$ and $a \notin Z(D)$ such that $a^{b}-a=c$. Then $b$ is in the normaliser in $D$ of $C_{D}(a)$ and $b \notin C_{D}(a)$. If $b^{n} \in Z(D)$ then the division ring generated by $C_{D}(a)$ and $b$ is noncommutative and finite-dimensional over the field $C_{D}(a)$, contradicting Corollary 4.7.

(ii) As $a \notin Z(D),(a+Z(D)) \cap Z(D)=\emptyset$. Lemma 5.3 (i) says there are only finitely many noncentral conjugacy classes in $D$ and so one of them, say $b^{D}$, must intersect $a+Z(D)$ in a generic subset of $a+Z(D)$ (namely a subset of $a+Z(D)$ of maximal $S U$-rank). Without loss of generality, $b \in a+Z(D)$. Thus 
$\left(b^{D} \cap(a+Z(D))-b\right)$ which equals $\left(b^{D}-b\right) \cap Z(D)$ is a generic subset of $Z(D)$ which is easily seen to be an additive subgroup, and thus an additive subgroup of finite index. It follows, as we are in characteristic 0 that $b^{D}-b$ contains $Z(D)$. In particular, note $a \in b+Z(D) \subset b^{D}$, so also $a^{D}-a$ contains $Z(D)$.

With the above lemmas we can finish the proof. We divide into cases.

Case I. $\operatorname{char}(D)=p>0$.

By Lemma 5.3(ii) and compactness there is a uniform bound, say $m$, on the index in $D^{+}$of the subgroup $\left\{x^{a}-x: x \in D\right\}$ for $a \in D \backslash Z(D)$. We will show that every element of $D^{*} / Z(D)^{*}$ has finite order, which by Kaplansky's Theorem (see 5.15 in [6]) implies that $D$ is commutative. If $C_{D}(a)$ has cardinality at most $m$ then clearly $a$ has exponent at most $m$ in $D$. Otherwise we can (by choice of $m$ ) find nonzero $a^{\prime} \in C_{D}(a)$ of the form $b^{a}-b$ for some $b \in D$. Note that $a$ and $b$ do not commute. But $a^{p}$ and $b$ do commute $\left(b^{a}=b+a^{\prime}\right.$ implies $\left.b^{a^{p}}=b+p a^{\prime}=b\right)$. So $a^{p}$ is in $C_{D}(a) \cap C_{D}(b)$ which by Lemma 5.4 equals $Z(D)$. So $a$ has order $p$ in $D^{*} / Z(D)^{*}$. This finishes the proof of Theorem 5.1 in the positive characteristic case.

Case II. $\operatorname{char}(D)=0$.

Suppose to begin with that every element of $Z(D)$ has a square root in $Z(D)$. Thus, we obtain infinitely many roots of unity $\left\{\omega_{i}: i<\omega\right\}$ in $Z(D)$. Let $a \notin Z(D)$. Then for some $i<j, \omega_{i} . a$ and $\omega_{j} . a$ are in the same conjugacy class (as by Lemma 5.3 (i) there are only finitely many noncentral conjugacy classes). Thus $a$ is conjugate to $\omega . a$ for some nontrivial root of unity $\omega$, say $a^{b}=\omega \cdot a$. Note then that $b \notin C_{D}(a)$, but $b^{m} \in C_{D}(a)$ for $m$ such that $\omega^{m}=1$. By Lemma $5.4, b^{m} \in Z(D)$. This contradicts Lemma 5.5 (i).

So we may assume that some $c \in Z(D)$ has no square root in $Z(D)$, and thus by Lemma 5.5 (i) $c$ is not a square in $D$. Choose $a \in D \backslash Z(D)$, and let $F$ $=C_{D}(a)$, a (supersimple) field containing $Z(D)$. Let $T=\left(F^{*}\right)^{2}$, which is by supersimplicity a definable subgroup of $F^{*}$ of finite index. Let $z \in Z(D)$ be nonzero. By Proposition 3.4 there are $b \in T$ and $e \in c . T$, both generic over $z$ in $F$ such that $b-e=z$. Note that $e$ is not a square in $D$ (as $c$ is not). As $e$ is generic in $F, e \notin Z(D)$ so by Lemma 5.5 (ii), $e^{d}-e=z$ for some $d \in D$. Thus $e^{d}=b$. But $b$ is a square, while $e$ and thus $e^{d}$ is not a square, contradiction. This completes the proof of Theorem 5.1, and also the paper.

\section{Acknowledgements}

The first author would like to thank Zoe Chatzidakis for many fruitful discussions around the time when he first began considering the issues dealt with in this paper. In particular the result that if $K$ is a supersimple field of characteristic 0 , then any rational curve over $K$ has a $K$-rational point, was obtained together with her. 
The result on the triviality of the Brauer group was obtained by all three authors, and the results in section 5 on division rings were obtained by Wagner.

\section{References}

1. G. Cherlin and S. Shelah, Superstable fields and groups, Annals of Math. Logic 18 (1980), 227-270.

2. N. Jacobson,Basic Algebra II, second edition. Freeman, 1989.

3. B. Kim, Forking in simple unstable theories, J. London. Math. Soc. (to appear).

4. B. Kim and A. Pillay, Simple theories, Ann. Pure Appl. Logic 88 (1997), 149-164.

5. E. Hrushovski, Pseudofinite fields and related structures, preprint 1991.

6. T. Y. Lam, A first course in noncommutative rings, Grad. Texts in Math, 118, SpringerVerlag.

7. A. J. Macintyre, On $\omega_{1}$-categorical theories of fields, Fund. Math. 71 (1971), 1-25.

8. A. Pillay, Definability and definable groups in simple theories, Jour. Symbolic Logic (to appear).

9. A. Pillay and B. Poizat, Corps et Chirurgie, Jour. Symbolic Logic 60 (1995), 528-533.

10. B. Poizat, Groupes Stables, Nur-al Mantiq wal-Ma'rifah, Villeurbanne, 1987.

11. J.-P. Serre, Local fields, Springer-Verlag, 1979.

12. S. Shelah, Simple unstable theories, Ann. of Math. Logic 19 (1980), 177-203.

13. F .O. Wagner, Groups in simple theories, preprint 1997.

Dept. of Math., Altgeld Hall, 1409 W. Green St, Urbana, IL 61801, USA

E-mail address: pillay@math.uiuc.edu

Dept. of Math., University of California, Berkeley, CA 94720, USA

E-mail address: scanlon@math.berkeley.edu

Maths. Institute, Oxford OX1 3LB, England

E-mail address: fwagner@maths.ox.ac.uk 